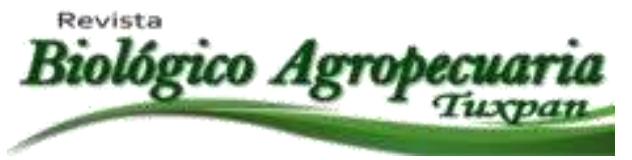

\title{
Diversidad taxonómica del ensamblaje de peces arrecifales en la costa oeste de la bahía de La Paz, BCS, México
}

Taxonomic diversity of reef fish assemblage on the west coast of the Bay of La Paz, BCS Mexico

$$
\text { Barjau-González E. }^{1 凶}, \text { J. Rodriguez-Romero² y F. Galvan-Magaña }{ }^{3}
$$

${ }^{1}$ Departamento Académico de Biología Marina, Universidad Autónoma de Baja California Sur (UABCS), Apdo. Postal 19-B, La Paz, Baja California Sur, C.P. 23080, México. ${ }^{2}$ Centro de Investigaciones Biológicas del Noroeste (CIBNOR), Apdo. Postal 128, La Paz, Baja California Sur, C.P. 23000, México. ${ }^{3}$ Centro Interdisciplinario de Ciencias Marinas (CICIMAR-IPN),

Departamento de Pesquerías y Biología Marina. Apdo. Postal 592. La Paz, Baja California Sur, México. C.P. 23000 México.

\section{${ }^{\bowtie}$ Autor para correspondencia: ebarjau@uabcs.mx}

Recibido: $24 / 08 / 2013$
Aceptado: 19/12/2013

\section{RESUMEN}

La Bahía de La Paz, es una de las bahías más grandes de la zona central del Golfo de California, esta zona se caracteriza por los altos niveles de captura comercial de peces que existen en la zona; sin embargo el número de investigaciones realizadas para conocer su diversidad taxonómica es bajo. Se determinó la variación en la diversidad taxonómica del ensamblaje de peces en ocho localidades de la zona oeste de la Bahía de La Paz, en el periodo de marzo de 2002 a abril de 2003. Se evaluaron los cambios en la diversidad de peces de arrecifes rocosos considerando la distancia taxonómica entre las especies, utilizando los índices de diversidad alfa, alfa promedio, beta y gamma, y los índices de distintividad taxonómica $\left(\Delta^{*}\right)$, así como la distintividad taxonómica promedio $(\Delta+)$.Se realizaron

censos visuales en 48 transectos de $100 * 5 \mathrm{~m}\left(500 \mathrm{~m}^{2}\right)$ a una profundidad promedio de $5 \mathrm{~m}$ en un horario de 09:00 a 16:00 horas. Se observaron 24,633 organismos pertenecientes a 92 especies de 67 géneros de peces. De acuerdo a los índices de diversidad Alfa Promedio, Beta y Gamma, el mes con mayor diversidad fue agosto (19.5, 40.5 y 60 especies, respectivamente) y el de menor diversidad fue diciembre (20.625, 27.375 y 48 especies, respectivamente). Los índices de distintividad taxonómica $\left(\Delta^{*}\right)$, y distintividad taxonómica promedio $(\Delta+)$, en el análisis espacial no mostraron diferencias $(\mathrm{p}=>0.05)$, mientras que en su forma temporal ambos mostraron diferencias $(\mathrm{p}=<0.05)$.

Palabras claves: Diversidad taxonómica, peces arrecifales, Bahía de La Paz. 


\begin{abstract}
La Paz bay, is one of the largest bays in the central zone of the Gulf of California, this area is characterized by high levels of commercial harvest of fish that exist in the area, but the number of investigations conducted for taxonomic diversity is low. We determined the variation in taxonomic diversity of coastal reef fish assemblage at eight locations along the west side of La Paz bay, during the period march 2002 to april 2003. We assessed changes in taxonomic diversity considering fish taxonomic distance between species diversity indices using alpha, average, beta and gamma, and taxonomic distinctness indices $(\Delta *)$ and the average taxonomic distinctness $(\Delta+)$. Visual censuses were conducted in 48 transects of $100 * 5 \mathrm{~m}(500 \mathrm{~m} 2)$ at an average depth of $5 \mathrm{~m}$ at a time of 9:00 to 16:00 hours. There were 24,633 organisms belonging to 92 species of 67 genera of fish. According to Alfa Average diversity indices, Beta and Gamma, the month with the highest diversity was August (19.5, 40.5 and 60 species, respectively) and the lowest diversity was in December (20,625, 27,375 and 48 species, respectively). Taxonomic distinctness indices $(\Delta *)$ and average taxonomic distinctness $(\Delta$ $+)$ in the spatial analysis showed no difference $(\mathrm{p}=<0.05)$, while in temporary form both showed differences $(\mathrm{p}=<.05)$.
\end{abstract}

Keywords: Taxonomic diversity, coastal reef fish, La Paz bay, visual censuses.

\section{INTRODUCCIÓN}

Para la zona costera de la Bahía de La Paz (ZCBP) no existen estudios que consideren la diversidad taxonómica de los peces de arrecifes rocosos. Solo se tienen estudios sobre la estructura de las comunidades de peces de la bahía, a pesar de tener una gran importancia desde el punto de vista pesquero, turístico y minero; además, la bahía es la entrada y salida de embarcaciones de diferentes calados a la ciudad y puerto de La Paz.

A partir de 1995, Warwick \& Clarke (1995, 1998) y Clarke \& Warwick (1998, 1999, 2001), propusieron cuatro índices de diversidad taxonómica que consideran las diferencias

taxonómicas entre especies. Estos cuatro índices se pretende que sean independientes del tamaño de la muestra, relacionados con la diversidad funcional y el impacto ambiental (Clarke \& Warwick 1998; Rogers et al., 1999; Clarke \& Warwick 2001; Warwick \& Clarke 2001; Warwick et al., 2002; Warwick \& Light 2002).

Para conocer como está conformada la diversidad taxonómica de la comunidad íctica de la zona costera oeste de la bahía de La Paz, se evalúan los cambios en la diversidad de peces de arrecifes rocosos basadas en la distancia taxonómica entre especies, utilizando los índices de distintividad taxonómica $\left(\Delta^{*}\right) \mathrm{y}$ distintividad taxonómica promedio $\left(\Delta^{+}\right)$.

\section{MATERIALES Y MÉTODOS}

Área de estudio

Revista Científica Biológico Agropecuaria Tuxpan 1 (2) 
La Bahía de La Paz está ubicada sobre el margen suroriental de la Península de Baja California, tiene una forma semielíptica con su eje mayor orientado en dirección nornoroestesursureste, está limitada hacia el oeste y sur por tierra firme, hacia el norte y oriente por las aguas del Golfo de California y las islas Espíritu Santo y Partida. Las localidades de muestreo (Fig. 1) se caracterizan por presentar una cobertura aproximada de $90 \%$ de arrecifes rocosos.

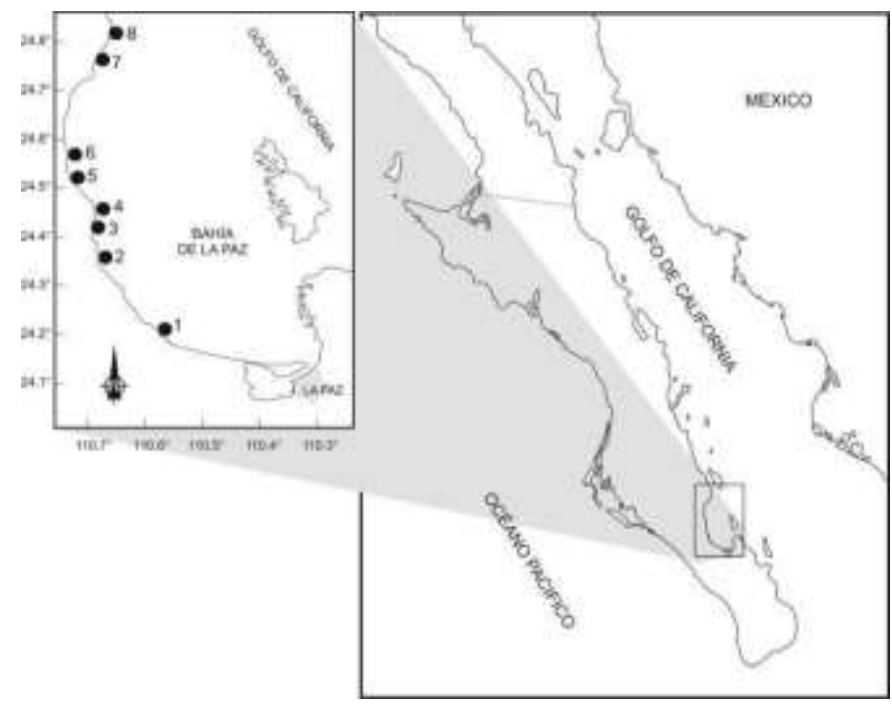

Figura 1. Mapa de las localidades muestreadas en la ZCBP: 1) Las Pacas, 2) Piedra Bola, 3) San Juan de la costa, 4) Estacuda, 5) El Cobre, 6) El Guano, 7) Portugués, 8) Mechudo.

Se realizaron seis campañas de muestreo bimestrales durante abril, junio, agosto, octubre y diciembre de 2002 y marzo 2003, en ocho localidades de la zona oeste de bahía de La Paz, realizándose un total de 48 censos visuales en todo el periodo de estudio para determinar la diversidad taxonómica de los peces. Se realizaron censos visuales en cada localidad por medio de buceo libre, utilizando transectos de 100 * 5 metros (Letorneur 1996; JiménezGutiérrez 1999; Rodríguez-Romero et al., 2005; Barjau et al., 2012). La profundidad promedio en la cual se realizaron los censos fue de 5 metros.
Para el análisis de la diversidad alfa, beta y gamma, se utilizaron los datos de presencia/ausencia y para la diversidad taxonómica se utilizaron los índices distintividad taxonómica $\left(\Delta^{*}\right)$ y la distintividad taxonómica promedio $\left(\Delta^{+}\right)$, para el primero, se utilizaron los datos de abundancia total, los cuales fueron estandarizados de acuerdo a Clarke \& Warwick (2001), mientras que en el segundo método se utilizaron los datos de presencia/ausencia.

Para el cálculo de estos dos últimos índices se utilizaron seis niveles jerárquicos (especies, género, familia, orden, clase y phyllum).

\section{Revista Científica Biológico Agropecuaria Tuxpan 1 (2)}




\section{RESULTADOS}

De acuerdo a los parámetros fisicoquímicos, la temperatura del agua fue el factor que presentó mayor variación en el área de estudio, se observaron dos temporadas climáticas: una fría (abril, marzo y junio) con temperatura promedio de $22.57^{\circ} \mathrm{C}$, y la otra cálida (agosto, octubre y diciembre) con temperatura promedio de $27.09^{\circ} \mathrm{C}$; es de importancia resaltar que el mes de diciembre se incluye en la temporada cálida, en virtud de que este mes registró una mayor temperatura $\left(24.26^{\circ} \mathrm{C}\right)($ Cuadro 1$)$.

Se registraron un total de 24,633 organismos, pertenecientes a 92 especies de 67 géneros y 40 familias. Las familias con más especies fueron: Pomacentridae (8), Labridae (7), Haemulidae (6), Serranidae (6), Scaridae (5), Lutjanidae (4) y Tetraodentidae (4).

De acuerdo al cálculo del índice de diversidad alfa por localidades y meses no mostraron diferencias estadísticas $(\mathrm{F}(7,40)=1.594, \mathrm{p}=0.1653)$ y $(\mathrm{F}(5,42)=0.5572$, $\mathrm{p}=0.7320$ ) respectivamente. En general, el comportamiento de este índice nos indica que no hubo un patrón definido, ya que en los meses cálidos sólo diciembre estuvo por arriba de la media general (20.62 especies), por otro lado, en los meses fríos, marzo obtuvo el mayor valor, los otros dos meses (abril y junio) estuvieron por debajo del promedio (Tabla 1).

En cuanto a las diversidades beta y gamma, en sus forma temporal, se registraron los mayores valores en el mes de agosto (41 y 60 especies), y los menores fueron en el mes de diciembre (27 y 48 especies) (Cuadro 1).

El índice de distintividad taxonómica $(\Delta$ *), calculándose con datos de abundancia total, mostró diferencias estadísticas $(\mathrm{F}(5,42)=5.845$, $\mathrm{p}=0.0003$ ). En relación a las localidades, no mostraron diferencias estadísticas ( $\mathrm{F}$ $(7,40)=0.5197, \mathrm{p}=0.8142$ (Cuadro 1$)$.

De acuerdo al índice de distintividad taxonómica promedio $(\Delta+)$, utilizando los datos de presencia/ausencia, mostró diferencia estadísticas $(\mathrm{F}(5,42)=3.7193, \mathrm{p}=0.0071)$ (Tabla 1). En relación a las localidades, no mostraron diferencias estadísticas (F $\quad(7,40)=1.5675$, $\mathrm{p}=0.1733)($ Cuadro 1$)$.

Cuadro 1. Relación de los parámetros físicos, químicos y taxonómicos por localidad, meses y temporadas climáticas: Temperatura $\left(\mathbf{T}^{\circ} \mathbf{C}\right)$, salinidad (S\%o), oxígeno disuelto (OD), Diversidad alfa $(\alpha)$, Diversidad alfa promedio $(\bar{\alpha})$, Diversidad beta $(\beta)$, Distintividad taxonómica $\left(\Delta^{*}\right)$ y Distintividad taxonómica promedio $(\Delta+)$.

\begin{tabular}{lcccccccc}
\hline Localidad/Mes/Temp. & $\mathbf{T}^{\mathbf{}} \mathbf{C}$ & $\mathbf{S \%}$ & $\mathbf{O D}$ & $\boldsymbol{\alpha}$ & $\overline{\boldsymbol{\alpha}}$ & $\boldsymbol{\beta}$ & + & $*$ \\
\hline Las Pacas & 24.30 & 35.08 & 5.74 & 35 & 13.83 & 21.167 & 59.292 & 55.835 \\
Piedra Bola & 24.95 & 35.02 & 5.70 & 46 & 18.16 & 27.833 & 56.568 & 53.73 \\
San Juan & 25.30 & 35.03 & 6.05 & 50 & 22 & 28 & 54.839 & 52.666 \\
Tarabilla & 25.08 & 35.02 & 6.26 & 43 & 19.5 & 23.5 & 55.735 & 54.154 \\
El Guano & 25.03 & 35.02 & 6.33 & 46 & 21.5 & 24.5 & 53.586 & 53.664 \\
El Cobre & 24.72 & 35.00 & 6.33 & 41 & 20 & 21 & 54.466 & 53.231 \\
Portugués & 24.45 & 34.98 & 5.74 & 49 & 20 & 29 & 55.846 & 52.994 \\
Mechudo & 24.30 & 35.08 & 5.26 & 44 & 19.16 & 24.833 & 55.798 & 53.694 \\
Abril & 22.63 & 35.03 & 3.68 & 55 & 18 & 37 & 56.159 & 53.989 \\
\hline
\end{tabular}


Barjau-González et al., 2014

\begin{tabular}{lcccccccc}
\hline Junio & 22.68 & 35.06 & 7.33 & 53 & 18.5 & 34.5 & 58.519 & 56.691 \\
Agosto & 29.63 & 35.09 & 5.70 & 60 & 19.5 & 40.5 & 54.481 & 52.176 \\
Octubre & 27.39 & 35.04 & 5.67 & 53 & 17.87 & 35.125 & 54.016 & 51.881 \\
Diciembre & 24.26 & 34.96 & 6.48 & 48 & 20.62 & 27.375 & 54.984 & 52.624 \\
Marzo & 22.41 & 35.00 & 6.71 & 54 & 21.12 & 32.875 & 56.663 & 54.749 \\
Temporada Cálida & 27.09 & 35.03 & 5.9 & 54 & 19.208 & 23 & 56.995 & 54.252 \\
Temporada Fría & 22.57 & 35.00 & 6.37 & 48 & 19.333 & 21.333 & 55.646 & 53.234 \\
\hline
\end{tabular}

\section{DISCUSIÓN}

La zona oeste de la Bahía de La Paz es de gran importancia comercial, por encontrarse una de las mineras más importantes del estado de BCS y por tener un sistema de cultivo de camarón, además que en este ecosistema se albergan una gran riqueza de especies de importancia ecológica, comercial y turística. Las especies de peces observadas en los sustratos duros de la ZCBP, incluye 92 especies de peces conspicuos, tanto permanentes como estacionales.

En otras áreas del Golfo de California como son Isla San José, Isla Cerralvo e Isla Espíritu Santo, así como la costa este de la bahía se ha documentado que la temperatura del agua $\mathrm{y}$ la diversidad en fauna $\mathrm{y}$ flora circundante en el sustrato arrecifal, contribuye a incrementar la abundancia y diversidad de peces, siendo una caracterización estructural que se manifiesta en otros ecosistemas tropicales y subtropicales del sur del Golfo de California (Jiménez-Gutiérrez 1999; Rodríguez-Romero et al., 2005; BarjauGonzález et al., 2012), han argumentado que la temperatura del agua, el tipo de hábitat, alimentación y zonas de crianza y protección que proporcionan los arrecifes rocosos contribuyen en gran medida a elevar la abundancia, concentración y riqueza específica de especies de peces (Pérez-España et al.,1996;
Galván-Piña et al., 2003; Dominici-Arosamena y Wolff 2006; Barjau-González et al., 2012).

Rodríguez-Romero et al., (1998,2005), Galván-Magaña et al., (2000) y Barjau et al., 2012 señalan que los cambios estructurales en la riqueza de especies durante temporadas pueden ser explicados con base en la temperatura del agua, el tipo de sustrato y flora circundante que se establece en forma diferencial dentro del Golfo de California. El número de trabajos publicados sobre la estructura comunitaria de la ictiofauna arrecifal conspicua que se han realizado en diversos ecosistemas del sur del Golfo de California, principalmente dentro de Bahías, islas, esteros y zonas arrecifales (Pérez España et al., 1996; Villareal-Cavazos et al., 2000; Aburto-Oropeza y Balart 2001; Viesca-Lobatón 2003; ÁlvarezFilip 2004; Campos-Dávila et al., 2005; Rodríguez-Romero 2005; Álvarez-Filip et al., 2006; Barjau-Gonzalez et al., 2012), han utilizado los índices de diversidad de Shannon, riqueza de especies y la equidad de Pielou, sin embargo, para la ZCBP no existen antecedentes de trabajos donde se utilicen los índice de diversidad alfa, beta y gamma, como componentes de la riqueza de especies, así como de los índices de distintividad taxonómica.

El índice de diversidad alfa, es considerado como el número de especies en un punto o en un sitio dado, en este sentido, no se presentaron diferencias estadísticas $(\mathrm{p}>0.05)$. 
La diversidad alfa refleja las relaciones ecológicas que se encuentran en un espacio puntual (Halffeter y Moreno 2005), lo que se infiere del porqué de esta variación entre localidades de la ZCBP.

La diversidad beta, mide las diferencias (el recambio) entre las especies de dos puntos, dos tipos de comunidad o dos paisajes, estas diferencias pueden ocurrir en el espacio, cuando las mediciones se hacen en sitios distintos en un mismo tiempo, o en el tiempo, cuando las mediciones se realizan en el mismo lugar pero en tiempos distintos (Halffeter y Moreno 2005); en este sentido, en la localidad El Portugués, se observó el mayor recambio de especies; mientras que en Las Pacas y el Cobre se observaron los menores recambios, esto coincide con lo considerado por Whittaker (1975) en el que claramente se refiere a las especies como entidades taxonómica y no a los números de especies. Por otro lado, Cornell y Lawton (1992), proponen que la diversidad beta es el enlace entre las diversidades alfa y gamma, por lo que nuestros resultados son coincidentes con lo observado por Koleff y Gastón (2002), los cuales midieron el spatial turnover (Diversidad beta) en términos de ganancia o pérdida de especies, lo que en este trabajo se presenta y documenta la influencia entre la diversidad beta y las diversidades alfa y gamma.

El índice de distintividad taxonómica promedio $\left(\Delta^{+}\right)$en su forma temporal mostró diferencias estadísticas $(\mathrm{p}=<0.05)$, obteniéndose el mayor valor en el mes de junio, lo que evidencia que las distancias taxonómicas promedio entre las especies no fueron muy cercanas taxonómicamente, esto pudiese ser explicado en que el mes de junio en esta zona es considerado como un mes de transición entre el verano y el otoño (Aguirre-Bahena, 2007).

En este estudio, al utilizar este índice en su forma espacial, no mostró diferencias estadísticas $(\mathrm{p}=>0.05)$, registrándose que la localidad denominada El Guano, está en el límite inferior del intervalo de confianza y que es la localidad que registró el menor valor, una explicación de esta observación, es que este sitio de alguna manera ha sido alterado antropogénicamente, esta observación coincide con lo reportado por Sosa-López et al., (2007), quienes realizaron un estudio en la Laguna de Términos, Campeche, y encuentran variación en la estructura taxonómica de la comunidad local y lo explican con base a tres factores: 1) actividad pesquera, 2) contaminantes, y 3) cambios en las condiciones hidrológicas.

Por otra parte, se puede considerar que las especies cercanas taxonómicamente utilizan hábitats y recursos semejantes. En este contexto Warwick y Clarke (2001) encontraron que $\Delta^{+}$ puede discriminar tipos de hábitats que tienen bajos valores de distintividad y que están cercanamente asociados con los niveles tróficos. La variación de $\Delta+$ entre localidades puede ser debido al bajo número de grupos tróficos.

\section{CONCLUSIONES}

Considerando la diferenciación taxonómica $\Delta^{*}$ por localidades de muestreo, excepto por la localidad El Guano, la cual mostró diferencias con respecto a las otras, infiriendo que posiblemente este sitio pudo haber estado bajo presión antropogénica.

La determinación del promedio de las ramas del árbol filogenético, mediante la diferenciación taxonómica promedio $\Delta+$, sugiere que hay dos localidades que se consideran como muy estables; mientras que el resto de las localidades se ubican muy cercanas al promedio y pueden considerarse estables, además de que las especies observadas no están muy relacionadas filogenéticamente. Se puede inferir que la localidad El Guano pudo haber sido impactada antropogénicamente y las especies observadas en esa localidad están más relacionadas filogenéticamente.

\section{Revista Científica Biológico Agropecuaria Tuxpan 1 (2) ISSN: 2007-6940}




\section{LITERATURA CITADA}

Aburto-Oropeza, O. y E.F. Balart. 2001. Community structure of reef fish in several habitats of a rocky reef in the Gulf of California.Marine Ecology. 22(4): 283-305.

https://doi.org/10.1046/j.1439-0485.2001.017 47.x

Aguirre-Bahena, F. 2007. Cambios temporales en los componentes y los flujos de la materia en hundimiento en la cuenca Alfonso, Bahía de La Paz, durante el periodo 2002-2005. Tesis doctoral. CICIMAR-IPN, 102 pp. https://doi.org/10.35537/10915/82130

Alvarez-Filip, L. and H. Reyes-Bonilla. 2006. Comparison of community structure and functional diversity of fishes at Cabo Pulmo coral reef, western Mexico between 1987 and 2003. Proceedings of

10th International Coral Reef

Symposium, 216-225.

https://doi.org/10.1111/j.1439-0485.2006.0010 8.x

Alvarez-Filip, L.; H. Reyes-Bonilla \& L.E. Calderon-Aguilera. 2006. Community structure of fishes in Cabo Pulmo Reef, Gulf of California. Marine Ecology 27: 253-262.

https://doi.org/10.1111/j.1439-0485.2006.0010 8.x

Barjau-González, E. ; J. Rodríguez-Romero; F. Galván-Magaña y J. López-Martínez. 2012. Estructura temporal y espacial de la comunidad de peces arrecifales de la Isla San José, Golfo de California, México. Revista Biología Tropical Vol. 60 (2): 649-667. https://doi.org/10.15517/rbt.v60i2.3957

Barjau-González, E. 2012. Estructura comunitaria y diversidad taxonómica de los peces en la bahía de la Paz y la Isla San José, Golfo de California. Tesis de Doctorado. CIBNOR. La Paz, B.C.S. México. 149p.

https://doi.org/10.15517/rbt.v60i2.3957
Barjau-González, E. ; J. Rodríguez-Romero; F. Galván-Magaña y J. López-Martínez. 2012. Changes in the taxonomic diversity of the reef fish community of San Jose Island, Gulf of California, Mexico. Biodiversity \&Conservation. 21:35433554. https://doi.org/10.1007/s10531-012-0378-z

Cornell, H.V. \& J.H. Lawton. 1992. Species interactions, local and regional processes and limits to the richness of ecological communities: A theorical perspective. Journal of Animal Ecology, 61:1-12. https://doi.org/10.2307/5503

Clarke, K.R. and R.M. Warwick. 1998. A taxonomic distinctness index and its statistical properties. Journal of Applied Ecology 35:523-531. https://doi.org/10.1046/j.1365-2664.1998.3540 523.x

Clarke, K.R. and R.M. Warwick. 1999. The taxonomic distinctness measure of biodiversity:weighting of step lengths between hierarchical levels. Marine Ecology Progress Series 184: 21-29. https://doi.org/10.3354/meps184021

Clarke, K.R. and R.M. Warwick. 2001. A further biodiversity index applicable to species lists: variation in taxonomic distinctness. Marine Ecology Progress Series 216: 265-278. https://doi.org/10.3354/meps216265

Dominici-Arosamena, A. and M. Wolff. 2006. Reef fish community structure in the Tropical Eastern Pacific (Panamá): living on a relatively stable rocky reef environment. Helgol Mar Res 60:287305. https://doi.org/10.1007/s10152-006-0045-4

Galván-Magaña. F.; F. Gutiérrez-Sánchez; L.A. Abítia-Cárdenas y J. Rodríguez-Romero. 2000. The distribution and affinities of the shore fishes of Baja California lagoons. Pages 338-398 In: M. Munawar, S. G. Lawrence, I.F. Munawar and D.F. Malley, (eds.) Aquatic Ecosystem of Mexico. Ecovision World 
Monograph series. Backhuys Publishers, Leiden, The Netherlands. https://doi.org/10.1086/378008

Galván-Piña, V. H.; F. Galván-Magaña; L. A. Abítia-Cárdenas; F. J. Gutiérrez-Sánchez y J. Rodríguez-Romero. 2003. Seasonal structure of fish assemblages in rocky and sand habitats in Bahía de La Paz, México. Bulletin of Marine Science. 72(1):19-35.

Halffter, G. \& C.E. Moreno. 2005. Significado Biológico de las diversidades Alfa, Beta y Gamma. En: G. Halffter, J. Soberón, P. Koleff \& A. Melic (eds.). Monografías Tercer Milenio, S.E.A., Zaragoza, España. Vol. 4:5-18.

Jiménez-Gutiérrez, S. V. 1999. Abundancia y estructura comunitaria de peces de arrecifes rocosos en la zona de Isla Cerralvo, B.C.S., Méx. Tesis de Maestría. CICIMAR. IPN. La Paz, B.C.S. México.

$91 \mathrm{pp}$.

https://doi.org/10.22201/ib.20078706e.2009.0 01.594

Koleff, P. \& K.J. Gaston. 2002. The relationships between local and regional species richness and species turnover. Global Ecology and Biogeography, 11: 363-375.

https://doi.org/10.1046/j.1466-822x.2002.003 02. $x$

Letourneur, Y.1996.Dynamic of fish communities on Reunion Fringing Reef, Indian Ocean. I Patterns of spatial fluctuation. J. Exp. Mar. Biol. Ecol.195: $1-30$. https://doi.org/10.1016/0022-0981(95)00089-5

Pérez-España, E. H.; F. Galván-Magaña y L. A. Abítia-Cárdenas. 1996. Variaciones temporales y espaciales en la estructura de la comunidad de peces de arrecifes rocosos del suroeste del Golfo de California, México. Ciencias Marinas. 22(3): 273-294.

https://doi.org/10.22201/ib.20078706e.2009. 001.594

Rodríguez-Romero, J.; L.A. Abítia-Cárdenas F. Galván-Magaña, J. Arvizu- Martínez y
P.B. Aguilar. 1998. Ecology of fish communities from the soft bottoms of Bahía Concepción, México. Archive of Fishery and Marine Research. 46(1): 6176.

Rodríguez-Romero. J.; A. F. Muhlia-Melo; F. Galván-Magaña; F.J. Gutiérrez-Sánchez y V. Gracia-López. 2005. Fish assemblages around Espiritu Santo Island and Espiritu Santo Seamount in the Lower Gulf of California, México. Bulletin of Marine Science. 64:383-390.

Rogers. S.I., Clarke; K.R. and J.D. Reynolds. 1999. The taxonomic distinctness of coastal bottom-dwelling fish communities of the North-east Atlantic. Journal of Animal Ecology 68,769-782. https://doi.org/10.1046/j.1365-2656.1999.003 27.x

Sosa-López, A.; J. Ramos-Miranda; D. FloresHernández; D. Mouillot y T. Do-Chi. 2005. Evidencia de Cambios en la Diversidad Ictiofaunística de la Laguna de Términos, México. JAINA Boletín Informativo, 15(1): 7-13.

Viesca-Lobaton, C. 2003. Cambios temporales en la estructura de la cominudad de peces de arrecifes rocosos en la parte suroccidental del Golfo de California. Tesis de licenciatura. UABCS. Mexico. La Paz, Mexico, 75 pp. https://doi.org/10.37543/oceanides.v23i1-2.4 3

Warwick, R.M. and K.R. Clarke. 1995. New biodiversity measures reveal a decrease in taxonomic distinctness which increasing stress. Marine Ecology Progress Series 129: 301-305. https://doi.org/10.3354/meps129301

Warwick, R.M. and K.R. Clarke. 1998. Taxonomic distinctness and environmental assessment. Journal of Applied Ecology 35: 532-543. https://doi.org/10.1046/j.1365-2664.1998.354 0532.x

Warwick, R.M. and K.R. Clarke. 2001. Practical measures of marine biodiversity 
based on relatedness of species. Oceanography \& Marine Biology Annual Review, 39,207-231.

Warwick, R.M.; C.M. Ashman; A.R. Brown; Clarke, K.R.; B. Dowell; B. Hart; R.E. Lewis; N. Shillabeer; P.J. Somerfield and J.F. Tapp. 2002. Inter-annual changes in the biodiversity and community structure of the macrobenthos in Tees Bay and the Tees estuary, UK, associated with local and regional environmental events. Marine Ecology Progress Series 234, 113. https://doi.org/10.3354/meps234001
Warwick, R.M. and J. Light. 2002. Death assemblages of molluscs on St Martin's Flats, Isles of Scilly, a surrogate for regional biodiversity? Biodiversity and Conservation 11: 99-112.

Whittaker, R.H. (Ed.) 1975. Communities and Ecosystems. MacMillan, New York. 385 pp.

Copyright (c) 2013 E. Barjau Gonzâlez, J. Rodriguez Romero yF. GalvánMagaña

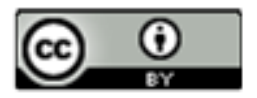

Este texto está protegido por una licencia Creative Commons 4.0

Usted es libre para Compartir —copiar y redistribuir el material en cualquier medio o formato- y Ad aptar el documento —remezclar, transformar y crear a partir del material- para cualquier propósito, inchso para fines comerciales, siempre que cumpla la condición de:

Atribución: Usted debe dar crédito a la obra original de manera adecuada, proporcionar un enlace a la licencia, e indicar si se han realizado cambios. Puede hacerlo en cualquier forma razonable, pero no de forma tal que sugiera que tiene el apoyo del licenciante olo recibe por el usoque hace de la obra.

Resumendelicencia $-\underline{\text { Textocompletodelalicencia }}$ 